\title{
Moisture Prediction from Simple Micrometeorological Data
}

\author{
Y. Chtioui, L. J. Francl, and S. Panigrahi
}

First and third authors: Agricultural and Bio-Systems Engineering Department, North Dakota State University (NDSU), Fargo 58105; and second author: Plant Pathology Department, NDSU. Accepted for publication 20 April 1999.

\section{ABSTRACT}

Chtioui, Y., Francl, L. J., and Panigrahi, S. 1999. Moisture prediction from simple micrometeorological data. Phytopathology 89:668-672.

Four linear regression methods and a generalized regression neural network (GRNN) were evaluated for estimation of moisture occurrence and duration at the flag leaf level of wheat. Moisture on a flat-plate resistance sensor was predicted by time, temperature, relative humidity, wind speed, solar radiation, and precipitation provided by an automated weather station. Dew onset was estimated by a classification regression tree model. The models were developed using micrometeorological data measured from 1993 to 1995 and tested on data from 1996 and 1997. The GRNN outperformed the linear regression methods in predicting moisture occurrence with and without dew estimation as well as in predicting

Moisture on plant surfaces has considerable influence on disease development in many economically important crops. The length of time that moisture is present is of major significance to lesion expansion of various fungal pathogens (18). Assessment of moisture occurrence and duration is beneficial for the individual growers as well as for the environment. For example, the estimation of moisture can provide a rational and efficient schedule for fungicide sprays. The chemical load on the environment, the labor, and the costs of agricultural production can then be reduced. For that purpose, some sensors have been designed for the direct measurement of wetness on leaves $(6,12,17)$. These sensors are often not appropriate because they require equipment maintenance and need to be installed in each individual farm. Prediction of moisture from micrometeorological factors supplied by automatic weather stations is advantageous since this can form the basis for a regional disease warning system $(5,11,13,14)$.

Models have been proposed to estimate dew duration as a means for forecasting disease development and intensity. A linear regression equation based on relative humidity, wind speed, and temperature was developed to predict dew duration (5). For tomato disease management, wetness duration was estimated as the length of time that the dew point depression remained between two specified limits (13). Gleason et al. (14) estimated duration of dew periods with a nonparametric classification procedure. The model predicted the absence or the presence of dew with a success rate of $83.5 \%$. An energy balance technique simulated the dew duration for exposed and shaded leaves $(25,26)$. The average dew duration was estimated within $60 \mathrm{~min}$ for shaded leaves. Francl and Panigrahi (8) illustrated that a back-propagation neural network could integrate hydric and other micrometeorological variables into a holistic method that was better in predicting the occurrence of moisture than stepwise logistic regression and multivariate discriminant analysis. This model predicted presence or absence of

Corresponding author: L. J. Francl; E-mail address: francl@badlands.nodak.edu

Publication no. P-1999-0601-01R

(C) 1999 The American Phytopathological Society duration of moisture periods. Average absolute error for prediction of moisture occurrence by GRNN was at least $31 \%$ smaller than that obtained by the linear regression methods. Moreover, the GRNN correctly predicted $92.7 \%$ of the moisture duration periods critical to disease development in the test data, while the best linear method correctly predicted only $86.6 \%$ for the same data. Temporal error distribution in prediction of moisture periods was more highly concentrated around the correct value for the GRNN than linear regression methods. Neural network technology is a promising tool for reasonably precise and accurate moisture monitoring in plant disease management.

Additional keywords: disease forecasting, leaf wetness, partial leastsquares regression, principal component regression, ridge regression.

moisture on leaves; however, prediction of a binary variable is inherently easier than prediction of a continuous one.

The generalized regression neural network (GRNN) automatically extracts the underlying (linear or nonlinear) regression surface from the available training data. Unlike other regression methods such as logistic or polynomial regression, the GRNN is a nonlinear (no linear assumption about the form of the regression model) and a nonparametric method (no assumption about the distribution of data in the regression model). The prediction is based on the computation of the most probable output value given a set of measured input patterns and their corresponding actual outputs. Outputs are predicted by a weighted sum of unit functions, which are centered at each training pattern. The width of each unit function is called the smoothing factor. Specht (30) proposed the "leave-one-out" method for the optimization of a single smoothing factor. Catasus et al. (2) attributed a random value to the smoothing factor and found that the GRNN was better than the multiple linear regression for modeling spectral inferences. Prediction accuracy may be improved by associating a smoothing factor with each input feature, but the computational time is markedly increased (3).

This paper considers the modeling of moisture occurrence and duration using readily available microclimatic data. Moisture prediction by the GRNN was compared with those obtained by four linear regression methods: multiple linear regression (MLR), ridge regression (RR), principal component regression (PCR), and partial least-squares regression (PLSR). These four linear regression methods have been applied extensively and successfully in many fields such as chemometrics (9), food science $(4,24)$, and chemistry (1). A desirable characteristic of the final, best model is an accuracy sufficient to permit its application in plant disease management.

\section{MATERIALS AND METHODS}

Data collection. Spring wheat (Triticum aestivum L.) was grown at the Agricultural Research Station of North Dakota State University in Fargo. In each year from 1993 to 1997, a block of approximately 0.7 ha was cultivated with customary agronomic practices. No fungicides were applied. Microclimatic measurements were recorded be- 
tween flag leaf emergence and the soft dough stage by an electronic datalogger (model CR10; Campbell Scientific, Logan, UT). Temperature, relative humidity, wind speed, solar radiation, and precipitation were measured every $10 \mathrm{~s}$ and summarized every 30 min by averaging temperature, relative humidity, and wind speed, and by summing solar radiation and precipitation. A feature "time" was included in the model to take into account the hour of the day.

Wetness was assessed by three to five replicate flat-plate resistance sensors (model 237; Campbell Scientific) that were placed at the flag leaf level of wheat. These sensors generated a resistance value indicative of wetness; data were collected every $120 \mathrm{~s}$ and averaged every $30 \mathrm{~min}$. The sensors were calibrated to generate a maximum resistance value of $10,000 \mathrm{k} \Omega$ (kilo-ohms) for a dry leaf surface (open-circuit). The resistance value was very low when the surface was wet (short-circuit). The moisture values were set to the range of zero to one by applying the logarithm (base 10) on the resistance values and dividing the result by four. Analysis of moisture duration requires that each period must be coded as dry or wet. A threshold indicating the transition between wet and dry surface was defined at a value of $900 \mathrm{k} \Omega$. Other researchers, using similar equipment, also defined a threshold of $900 \mathrm{k} \Omega$ (14).

Dew is a weather parameter of primary significance to disease development. In this study, dew formation was a microenvironmental factor for moisture prediction. The importance of dew to the agricultural community, together with the absence of dew measurements at standard weather stations, has resulted in the development of a set of models for dew estimation $(5,11,14,25,26)$. In this work, the empirical model proposed by Gleason et al. (14) was used since it provided $83.5 \%$ correct estimation of dew occurrence when tested in five midwestern states.

The data set containing seven features and moisture was split into two subsets: the training for model development and the test set for model validation. The training set included 3,503 observations from 1993, 1994, and 1995, while the test set comprised 2,256 observations gathered during 1996 and 1997. Prior to the application of any regression method, the training and test sets were mean centered and standardized (unit standard deviation for each of the seven features). Thus, all seven features had the same range of variation.

Linear regression methods. We assume that there are $n$ training patterns. Each pattern $x$ is represented by a set of measurements $x_{1}, x_{2}, \ldots, x_{p}$, in which $p$ refers to the number of features. A moisture value $y_{i}$ is associated with each pattern. Let $X$ denote the whole scaled (mean-centered and standardized) measured training data and $y$ the corresponding vector of moisture values.

Multiple linear regression (MLR). The MLR (also called least squares estimation) is based on the assumption that there is a linear relationship between the independent data (measured data) and the dependent data (data to be predicted),

$$
y=X \beta
$$

in which the vector $\beta$ represents the linear coefficients. It was demonstrated that the $\beta$ vector that minimizes the mean squared error in the predicted values of $y$ is defined by (28)

$$
\beta=\left(X^{t} X\right)^{-1} X^{t} y
$$

in which $X^{t}$ refers to $X$ transposed.

Ridge regression $(R R)$. The $\mathrm{RR}$ was introduced as an alternative to MLR in the presence of high correlations among the measured features (15). It is one of several biased regression methods. In this technique, the estimator of the regression coefficients is replaced by the equation

$$
\beta=\left(X^{t} X+k I\right)^{-1} X^{t} y
$$

in which $I$ refers to the identity matrix (diagonal elements of values 1). The RR, therefore, is based on the addition of a constant $k$ to the diagonal elements of the variance-covariance matrix $X^{t} X$ before inverting for least squares estimation. If $k=0$, we obtain the MLR. This procedure enhances the chances of getting a better matrix inversion in presence of a highly correlated data. Many estimators of $k$ have been proposed: the "ridge trace" (15), the "HoerlKennard-Baldwin" estimator (16), the "nonstochastic index of stability" (31), and the "variance inflation factors" estimator (28). In this study, the Hoerl-Kennard-Baldwin estimator was used. It has been mentioned prominently in the literature and fared well in many simulation studies (28).

Principal component regression (PCR). Like the RR, the goal of the PCR is to solve collinearity problems that may exist in the original measured data. The set of the measured features $(X)$ is transformed into a new set of uncorrelated and orthogonal features using principal component analysis (20). The PCR achieves the regression of the original dependent data $y$ on selected principal components of $X(21)$.

The rationale of the PCR is to work with the matrix of the principal component scores, rather than with the initial matrix $X$. In this study, the number of components for the PCR was selected by cross-validation. Let $c$ denote the number of principal components in the model and let $\hat{y}_{c}(i)$ denote the predicted moisture using $c$ principal components but not using the $i$ th pattern. The selected number of components is the one that minimizes

$$
\sum_{i=1}^{n}\left[y_{i}-\hat{y}_{c}(i)\right]^{2}
$$

Partial least-squares regression (PLSR). In PCR, the principal components associated with the measured data $X$ are generated in a manner that does not reflect the relationship between independent and dependent data. The PLSR introduces the linear relationship between independent and dependent data while iteratively extracting the components (32). The PLSR tries to create new factors that account for as much variation as possible. It is thus equivalent to the MLR, in which the set of predictors is replaced by a (usually smaller) set of components. Geladi and Kowalski (10) presented a detailed description of the PLSR algorithm. In the current study, the number of components was selected by analyzing the amount of residual variation (i.e., the norm of the vector of residuals). Addition of components stopped when introduction of a new component did not significantly decrease the norm of the vector of residuals.

Generalized regression neural network. The GRNN takes its basic concept from the Nadaraya-Watson kernel regression (22), which was mapped into a neural network $(29,30)$. The regression of a dependent feature $y$ on an independent set of features $X$ is the computation of the most likely value of $y$ given a set of measured $x$ and $y$,

$$
\hat{y}(x)=\frac{\sum_{i=1}^{n} y_{i} e^{-\sum_{j=1}^{p}\left(\frac{x_{j}-x_{i j}}{\sigma}\right)^{2}}}{\sum_{i=1}^{n} e^{-\sum_{j=1}^{p}\left(\frac{x_{j}-x_{i j}}{\sigma}\right)^{2}}}
$$

in which $\sigma$ represents the width of the unit function, here specified by the Gaussian function. In this work, a single smoothing factor $\sigma$ was associated with the whole data set and was optimized using an empirical method

$$
\sigma=\frac{s}{n^{\frac{1}{2 p}}}
$$

in which the parameter $s$ is called a scale factor (27). The objective function to be minimized was the mean square error for the prediction of the training set by cross-validation (3).

Model evaluation. The prediction performances were analyzed by the mean square error and the average absolute error. Mean square error is the average sum of squares between the actual and the predicted moisture values. Average absolute error is the average sum of the absolute difference between the actual and the predicted moisture values. Further regression diagnostics were considered too detailed for the objectives of this study. 
For additional evaluation of model predictions, four wetness duration intervals were defined in relation to the risk of disease development. Categorization was based on mobile bioassay data collected for wheat diseases in the same location and time period (7). The goal was to quantify the actual and the predicted moisture duration to disease-related intervals:

0.5 to $5 \mathrm{~h}$ : no or very little disease.

5.50 to $11 \mathrm{~h}$ : conducive to aerially dispersed diseases and those with a short infection period requirement.

11.50 to $17 \mathrm{~h}$ : greater disease severity, possible shift in disease spectrum.

$17.50 \mathrm{~h}$ or more: conducive to splash-dispersed spores but not to desiccation of spore abscission layer and airborne dispersal.

All the five regression methods developed in this paper were implemented on a $\mathrm{PC}$ using $\mathrm{C}++$ programs (Borland $\mathrm{C}++$, version 5; Borland International Inc., Scotts Valley, CA). The matrix inversion operation (needed in MLR, RR, and PCR) was achieved using GaussJordan method (27). The scale factor of the GRNN (equation 6) was optimized between 0.0 and 10.0 using the Brent method (27).

\section{RESULTS AND DISCUSSION}

An important aspect in prediction problems is to determine the degree of redundancy or correlation in the measured independent data. Table 1 shows the correlation matrix of the seven input fea- tures. The maximum coefficient of correlation was -0.61 between solar radiation and relative humidity. Thus, available quantitative features were not highly correlated. This means that each feature includes information different from that provided by the other features. However, this does not imply that the available quantitative features were pertinent for prediction of moisture.

Prediction of moisture occurrence. Five regression methods were compared for the prediction of moisture occurrence (Table 2 ). The PCR results were provided using four components because the introduction of the fifth principal component did not significantly decrease the prediction error for the training set. Explicitly, introduction of the fifth principal component decreased the prediction error of the training set by cross-validation by $2.79 \mathrm{E}-05\left(<10^{-4}\right.$, which was the threshold $)$ in comparison to four components. Analogously, five components were used for the PLSR since the introduction of more than five components did not substantially decrease the norm of the vector of residuals for the dependent data. The four linear regression methods provided approximately the same prediction rates for both the training and test sets (Table 2). In both data sets, the GRNN outperformed the linear methods in predicting moisture occurrence. An average absolute error half that of the four linear regression methods was achieved on training cases. The accuracy of moisture prediction was reduced when the GRNN was applied to the test set, but absolute error was still at least $31 \%$ less than with other methods.

TABLE 1. Correlations among the independent variables for prediction of moisture occurrence and duration in a wheat field environment $(n=3,503)$

\begin{tabular}{|c|c|c|c|c|c|c|c|}
\hline Variable $^{a}$ & $\begin{array}{l}\text { Time } \\
\text { (h) }\end{array}$ & $\begin{array}{c}\text { Temperature } \\
\left({ }^{\circ} \mathrm{C}\right)\end{array}$ & $\begin{array}{l}\text { Relative humidity } \\
\qquad(\%)\end{array}$ & $\begin{array}{l}\text { Wind speed } \\
\qquad(\mathrm{m} / \mathrm{s})\end{array}$ & $\begin{array}{l}\text { Solar radiation } \\
\qquad\left(\mathrm{J} / \mathrm{m}^{2}\right)\end{array}$ & $\begin{array}{l}\text { Precipitation } \\
(\mathrm{mm})\end{array}$ & Dew \\
\hline Time & 1.00 & 0.39 & -0.40 & 0.14 & 0.18 & 0.01 & -0.36 \\
\hline Temperature & 0.39 & 1.00 & -0.61 & 0.10 & 0.56 & -0.06 & -0.40 \\
\hline Relative humidity & -0.40 & -0.61 & 1.00 & -0.22 & -0.61 & 0.15 & 0.56 \\
\hline Wind speed & 0.14 & 0.10 & -0.22 & 1.00 & 0.22 & 0.13 & -0.52 \\
\hline Solar radiation & 0.18 & 0.56 & -0.61 & 0.22 & 1.00 & -0.10 & -0.40 \\
\hline Precipitation & 0.01 & -0.06 & 0.15 & 0.13 & -0.10 & 1.00 & -0.01 \\
\hline Dew & -0.36 & -0.40 & 0.56 & -0.52 & -0.40 & -0.01 & 1.00 \\
\hline
\end{tabular}

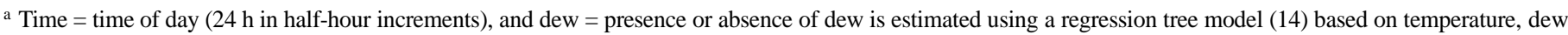
point depression, wind speed, and relative humidity.

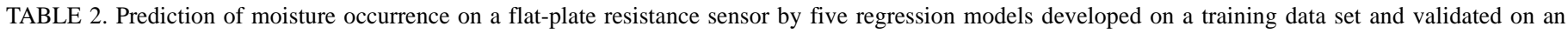
independent test data set

\begin{tabular}{|c|c|c|c|c|}
\hline \multirow[b]{2}{*}{ Method } & \multicolumn{2}{|c|}{ Training $(3,503 \text { patterns })^{\mathrm{a}}$} & \multicolumn{2}{|c|}{ Test $(2,256 \text { patterns })^{b}$} \\
\hline & Mean square error & Average absolute error & Mean square error & Average absolute error \\
\hline Multiple linear regression & 0.0219 & 0.1152 & 0.0237 & 0.1223 \\
\hline Ridge regression & 0.0219 & 0.1152 & 0.0237 & 0.1222 \\
\hline Principal component regression & 0.0228 & 0.1228 & 0.0249 & 0.1303 \\
\hline Partial least-squares regression & 0.0219 & 0.1153 & 0.0238 & 0.1224 \\
\hline Generalized regression neural network & 0.0122 & 0.0584 & 0.0221 & 0.0847 \\
\hline
\end{tabular}

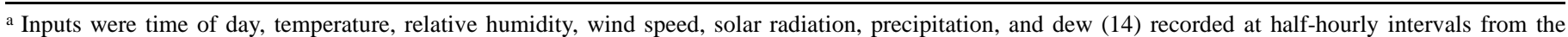
1993, 1994, and 1995 growing seasons. This data set was used to develop prediction equations and estimate error.

b Identical variables as in the training data set from the 1996 and 1997 growing seasons. These data independently validated the model estimates of error.

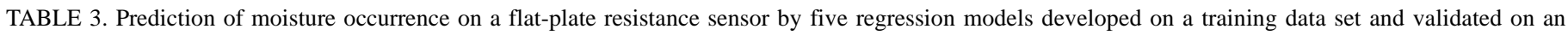
independent test data set without the empirical feature related to dew ${ }^{\mathrm{a}}$

\begin{tabular}{|c|c|c|c|c|}
\hline & \multicolumn{2}{|c|}{ Training set ${ }^{\mathrm{b}}$} & \multicolumn{2}{|c|}{ Test set ${ }^{\mathrm{c}}$} \\
\hline & Mean square error & Average absolute error & Mean square error & Average absolute error \\
\hline Multiple linear regression & 0.0251 & 0.1335 & 0.0303 & 0.1478 \\
\hline Ridge regression & 0.0251 & 0.1335 & 0.0303 & 0.1477 \\
\hline Principal component regression & 0.0256 & 0.1344 & 0.0300 & 0.1450 \\
\hline Partial least-squares regression & 0.0251 & 0.1336 & 0.0304 & 0.1480 \\
\hline Generalized regression neural network & 0.0115 & 0.0578 & 0.0219 & 0.0845 \\
\hline
\end{tabular}

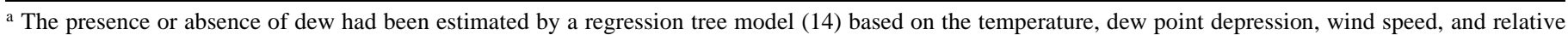
humidity.

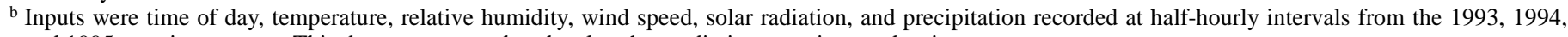
and 1995 growing seasons. This data set was used to develop the prediction equations and estimate error.

c Identical variables as in the training data set from the 1996 and 1997 growing seasons. These data independently validated the model estimates of error. 
In this study, the RR, PCR, and PLSR did not provide any improvement over MLR. This was because the input data consisted of largely uncorrelated features. The PCR and the PLSR were weak in extracting new underlying predictors that account for
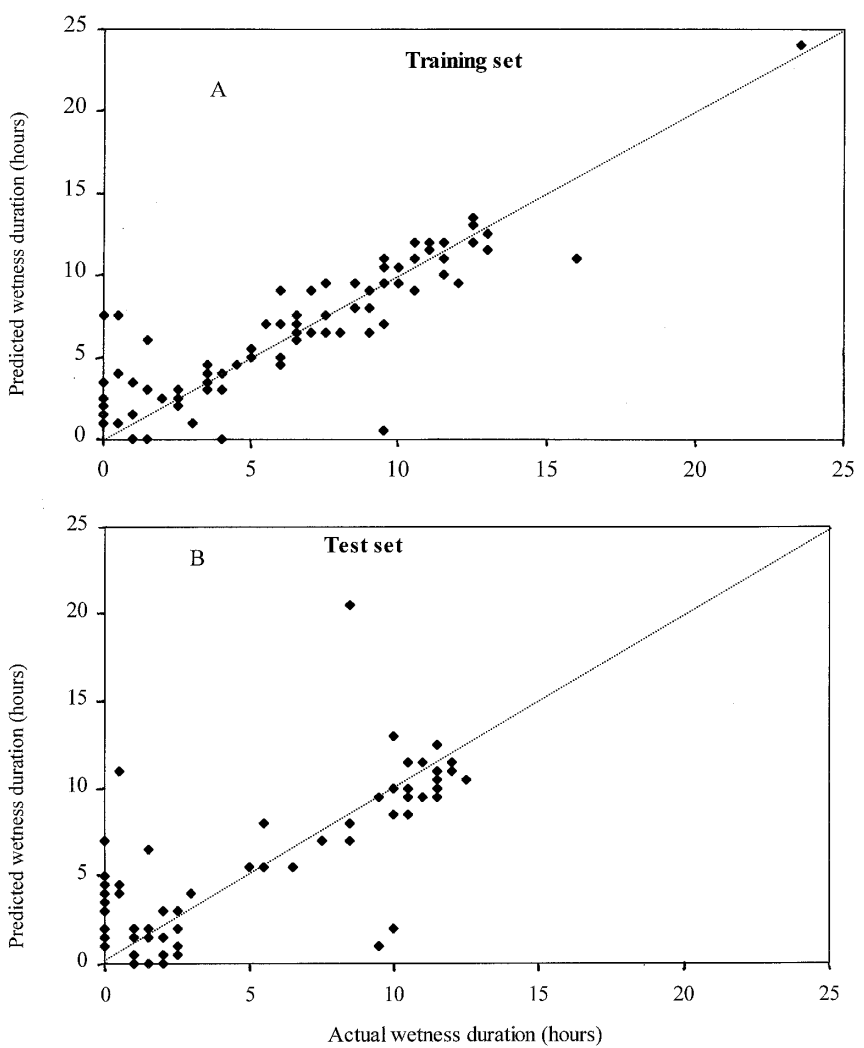

Fig. 1. Predicted versus measured moisture duration (h) using the generalized regression neural network: $\mathbf{A}$, training data for model development, and $\mathbf{B}$, test data for model validation. The number of points in these figures is equivalent to the number of moisture periods. most of the variation from uncorrelated measured data. The main limitation of the four linear regression methods is that they construct the regression surfaces assuming a linear relationship between the measured data and the data to be predicted. The GRNN is a nonlinear (no linear assumption about the relation between the variable to be predicted and the measured variables) and a nonparametric regression method. Additional analytical methods were available if wetness had been expressed in a binary rather than continuous fashion. For example, logistic regression on a similar data set produced an $88 \%$ accuracy rate on test data coded as wet $=1$ and dry $=0$ (8). However, a binary prediction is inherently easier to solve since the output variable has only two possible values.

Effect of dew. The prediction accuracies for the training set were extracted with and without the empirical feature "dew" (Tables 2 and 3). Taking into account dew slightly ameliorated the subsequent performance of the linear methods. For example, it decreased the average absolute error for the MLR by $13.7 \%$ for the training set. However, the feature dew did not improve the prediction of moisture

TABLE 4. Performance of five regression models in predicting the correct moisture duration period ${ }^{\mathrm{a}}$

\begin{tabular}{lcc}
\hline & $\begin{array}{c}\text { Training data }^{\mathrm{b}} \\
(\% \text { correct })\end{array}$ & $\begin{array}{c}\text { Test data }^{\mathrm{c}} \\
(\% \text { correct })\end{array}$ \\
\hline Multiple linear regression & 78.4 & 83.1 \\
Ridge regression & 78.4 & 83.1 \\
Principal component regression & 85.0 & 86.6 \\
Partial least-squares regression & 78.4 & 83.1 \\
Generalized regression neural network & 92.2 & 92.7 \\
\hline
\end{tabular}

${ }^{a}$ Defined as correctly categorizing wetness duration periods of 0 to $5 \mathrm{~h}$ and $5.5 \mathrm{~h}$ or more. Wetness duration was based on replicate flat-plate resistance sensor output. No error was registered if actual and predicted moisture duration belong to the same interval.

${ }^{\mathrm{b}}$ Inputs were time of day, temperature, relative humidity, wind speed, solar radiation, precipitation, and dew (14) recorded at half-hourly intervals from the 1993, 1994, and 1995 growing seasons. This data set was used to develop prediction equations and estimate error.

${ }^{c}$ Identical variables as in the training data set from the 1996 and 1997 growing seasons. These data independently validated the model estimates of error.
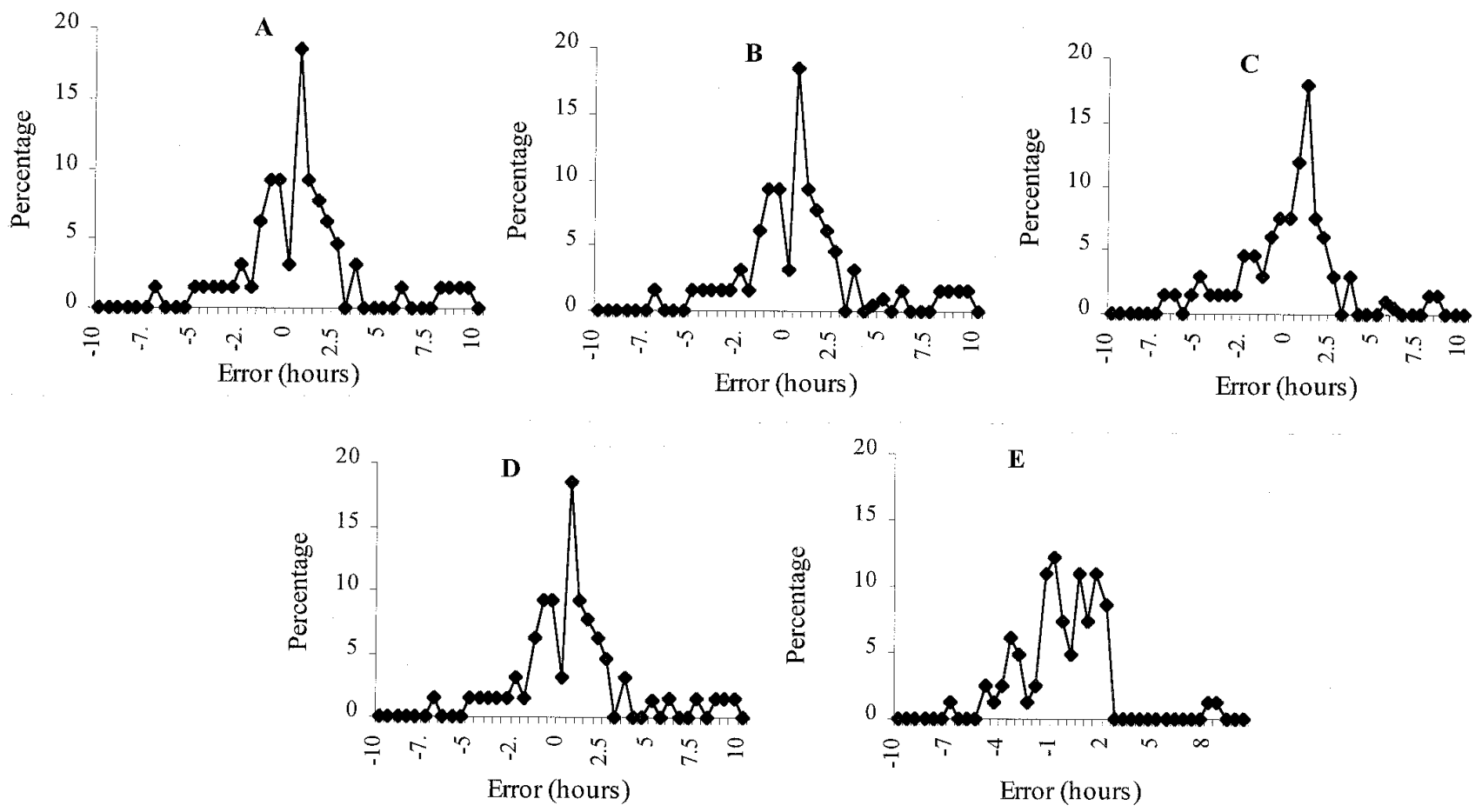

Fig. 2. Distribution of error values from five regression models that predicted moisture duration period: A, multiple linear regression, B, ridge regression, $\mathbf{C}$, principal component regression, $\mathbf{D}$, partial least-squares regression, and $\mathbf{E}$, generalized regression neural network. The error is the difference between independent test (validation) data and predicted moisture duration periods. Each point represents the percentage of moisture periods that were predicted with a specific error, and graphs were limited to between -10 and $+10 \mathrm{~h}$ error to eliminate outliers. 
occurrence by the GRNN. In this study, dew was empirically modeled by a nonlinear relationship of the temperature, the relative humidity, and the wind speed (14). The GRNN can implicitly extract nonlinear relationships from measured features, which may explain why introduction of the feature dew was not necessary for GRNN. It should be pointed out that the model that estimated dew did not take into account soil moisture and needs additional refinement. Modeling dew occurrence requires accurate estimation of dewfall (flux of water vapor coming from the atmosphere) and distillation (flux of water vapor coming from the ground) (23). The contribution of distillation in dew formation can be important and depends on soil moisture.

Prediction of moisture duration. Moisture duration directly influences development of pathogens on plants. Actual versus predicted moisture duration by GRNN was plotted for the training and test sets (Fig. 1A and B). The neural network model closely predicted moisture duration since most values were near the diagonal line. However, short moisture periods were slightly more difficult to predict than long ones. In this work, moisture duration was split into four intervals related to development of disease on wheat ( 0.5 to 5 , 5.5 to $11,11.5$ to 17 , and $17.5+\mathrm{h}$ ). No error was registered if actual and predicted moisture duration belong to the same interval. The accuracy of predicting potential disease development was extracted for the training and test sets (Table 4). MLR and RR provided identical performances, while PCR showed the best prediction accuracy of the linear methods. GRNN outperformed PCR by 7.1 and $6.1 \%$ in estimating biologically meaningful moisture duration periods for the training and test sets, respectively. Moisture on wheat leaves, therefore, can be estimated within informative categories from standard weather station observations with an average error of $8 \%$.

The distribution of errors in predicting moisture duration was analyzed for the test set, and the percentage of intervals associated with each error value was extracted (Fig. 2). The distribution of errors for GRNN was narrower than for other regression methods and was approximately symmetrical. The linear methods resulted in error distributions skewed toward underestimation (actual moisture periods exceeded predicted ones). Moisture periods were estimated within 2 h by GRNN $76 \%$ of the time and by PCR $72 \%$. Hence, an important proportion of errors (overestimations or underestimations) did not exceed $2 \mathrm{~h}$ for GRNN. The GRNN, therefore, provided better precision than the four linear regression methods. This result is significant because it provides a basis for a reasonable assessment of infection periods.

Although moisture prediction is a difficult task, good prediction results were obtained by the neural network approach. The performance of the GRNN method may have been due to the large number of input patterns available for training. Each node in the pattern layer of the GRNN represents a training pattern. In extremely large data sets, proliferation of these nodes can be reduced by using a clustering procedure (19). Patterns that are close in the feature space can be gathered to generate persistent new patterns.

Moisture on leaves exhibits spatial variability because microenvironmental factors vary according to the architecture and spatial characteristics of leaves, i.e., density, size, shape, inclination, and height. Moreover, micrometeorological conditions are nonstationary and may be climatically unique. Therefore, further research is needed to test the general applicability of the GRNN moisture model in other sites and crops. This will allow evaluation of the flexibility of the presented neural network-based regression model in case of location-specific environmental factors such as mist, fog, or prolonged high humidity. The neural network-based model presented here is promising but needs to be tested under other conditions before hardware or software integration into a pest management system.

\section{ACKNOWLEDGMENTS}

This work was funded in part by a grant (no. 9501094) from the USDA-CSREES National Research Initiative. We thank C. Doetkott, research analyst at NDSU, for statistical consultations.

\section{LITERATURE CITED}

1. Brakstad, F., Karstang, T. V., Sorensen, J., and Steen, A. 1988. Prediction of molecular weight and density of distillation fractions from gas chromatographic-mass spectrometric detection and multivariate calibration. Chemometrics Intelligent Lab. Syst. 3:321-328.

2. Catasus, M., Branagh, W., and Salin, E. D. 1995. Improved calibration for inductively coupled plasma-atomic emission spectrometry using generalized regression neural networks. Appl. Spectrosc. 49:798-807.

3. Chtioui, Y., Panigrahi, S., and Francl, L. A generalized regression neural network and its application to forecast plant disease. Chemometrics Intelligent Lab. Syst. In press.

4. Chtioui, Y., Panigrahi, S., and Marsh, R. 1998. Conjugate gradient and approximate Newton methods for an optimal probabilistic neural network for food color classification. J. Opt. Eng. 37:3015-3023.

5. Crowe, M. J., and Coakley, S. M. 1978. Forecasting dew duration at Pendleton, Oregon, using simple weather observations. J. Appl. Meteorol. 17:1482-1487.

6. Davis, D. R., and Hughes, J. E. 1970. A new approach to recording the wetting parameter by the use of electrical resistance sensors. Plant Dis. Rep. 54:474-479.

7. Francl, L. J. 1995. Challenge of bioassay plants in a monitored outdoor environment. Can. J. Plant Pathol. 17:138-143.

8. Francl, L. J., and Panigrahi, S. 1997. Artificial neural network models of wheat leaf wetness. Agric. For. Meteorol. 88:57-65.

9. Frank, I. E., and Friedman, J. H. 1993. A statistical view of some chemometrics regression tools. Technometrics 35:109-135.

10. Geladi, P., and Kowalski, B. 1986. Partial least-squares regression: A tutorial. Anal. Chim. Acta. 185:1-17.

11. Gillespie, T. J., and Barr, A. 1984. Adaptation of a dew estimation scheme to a new crop and site. Agric. Meteorol. 31:289-295.

12. Gillespie, T. J., and Kidd, G. E. 1978. Sensing duration of leaf moisture retention using electrical impedance grids. Can. J. Plant Sci. 58: 179-187.

13. Gillespie, T. J., Sristava, B., and Pitblado, R. E. 1993. Using operational weather data to schedule fungicide sprays on tomatoes in southern Ontario, Canada. J. Appl. Meteorol. 32:567-573.

14. Gleason, M. L., Taylor, S. E., Loughin, T. M., and Koehler, K. J. 1994. Development and validation of an empirical model to estimate the duration of dew periods. Plant Dis. 78:1011-1016.

15. Hoerl, A. E., and Kennard, R. W. 1970. Ridge regression: Biased estimation for nonorthogonal problems. Technometrics 12:55-67.

16. Hoerl, A. E., Kennard, R. W., and Baldwin, K. F. 1975. Ridge regression: Some simulations. Commun. Stat. A4:105-123.

17. Huband, N. D., and Butler, D. R. 1984. A comparison of wetness sensors for use with computer or microprocessor systems designed for disease forecasting. Proc. Br. Crop Prot. Conf. Pests Dis. 2:633-638.

18. Huber, L., and Gillespie, T. J. 1992. Modeling leaf wetness in relation to plant disease epidemiology. Annu. Rev. Phytopathol. 30:553-577.

19. Jain, A. K., and Dubes, R. C. 1988. Algorithms for Clustering Data. Prentice Hall, Englewood Cliffs, NJ.

20. Jolliffe, I. T. 1986. Principal Component Analysis. Springer-Verlag, New York.

21. Massy, M. F. 1965. Principal components regression in exploratory statistical research. J. Am. Stat. Assoc. 60:234-256.

22. Nadaraya, E. A. 1964. On estimating regression. Theory Probab. Its Appl. 10:186-190.

23. Oke, T. R. 1987. Boundary Layer Climates. 2nd ed. Routledge, New York.

24. Panigrahi, S., and Wiesenborn, D. 1994. Computer-based neuro-vision system for color classification of french fries. Pages 204-217 in: Proc. Opt. Agric. For. Biol. Proc. SPIE 2345.

25. Pedro, M. J., and Gillespie, T. J. 1982. Estimating dew duration. IUtilizing micrometeorological data. Agric. Meteorol. 25:283-296.

26. Pedro, M. J., and Gillespie, T. J. 1982. Estimating dew duration. IIUtilizing standard weather station data. Agric. Meteorol. 25:297-310.

27. Press, W. H., Teukolsky, S. A., Vetterling, W. T., and Flannery, B. P. 1992. Numerical Recipes in C. 2nd ed. Cambridge University, Cambridge, MA.

28. Ryan, T. P. 1997. Modern Regression Methods. John Wiley \& Sons, New York.

29. Schioler, H., and Hartmann, U. 1992. Mapping neural network derived from the Parzen window estimator. Neural Networks 5:903-909.

30. Specht, D. F. 1991. A general regression neural network. IEEE Trans. Neural Networks 2:568-576.

31. Vinod, H. D. 1976. Application of a new ridge regression method to a study of Bell system scale economies. J. Am. Stat. Assoc. 71:835-841.

32. Wold, H. 1985. Partial least squares. Pages 581-591 in: Encyclopedia of Statistical Science, Vol. 6. S. Kotz and N. Johnson, eds. John Wiley \& Sons, New York. 